\title{
Educacion Dual: Su Analisis Y Desarrollo Del Modelo Aleman Para Su Implementacion En El Entorno Laboral
}

\author{
Ibarra Mota Miriam \\ Bribiescas Silva Francisco Arturo \\ Departamento de Ciencias Sociales y Administración, \\ Universidad Autónoma de Ciudad Juárez, Mexico
}

Doi:10.19044/esj.2019.v15n4p143～ＵRL:http://dx.doi.org/10.19044/esj.2019.v15n4p143

\section{Resumen}

El presente artículo hace una investigación del sistema de educación dual alemán, así como su composición, implementación y resultados, así mismo presenta algunos casos de desarrollo en otros países y como este ha influenciado en algunos sistemas educativos alrededor del mundo. La educación es un factor clave para el desarrollo de todo país, pues juega un papel crítico en la productividad de sus mercados y en su crecimiento económico, debido a esto es de suma importancia los adecuados diseños de los modelos educativos desde su nivel más básico hasta su aspecto superior, así como el proporcionar una orientación vocacional funcional a todos aquellos que se encuentren próximos a entrar a la formación profesional. De esta manera se presenta la educación dual, un sistema probado a lo largo de los años en Alemania y otros países de Europa para formar a su fuerza laboral dentro de su campo de trabajo, interactuando en su entorno para aplicar lo aprendido. Este articulo analizará, mediante una investigación explicativa no experimental el desarrollo e implementación del sistema de educación dual alemán y como este ha impactado en el desempleo juvenil en su país natal, así como los casos registrados dentro de México y cuales han sido sus resultados a la fecha. Para esto se consultarán diversas fuentes de información que correspondan a casos mencionados en dichos países. El concepto de educación dual nace para dar respuesta a la necesidad de adecuar la Formación Profesional a las necesidades reales del mercado laboral, formando profesionales competentes dentro de su campo para que estos contribuyan de manera activa en las diferentes áreas de la industria ya sea pública o privada, siendo competentes y productivos en su especialidad, repercutiendo al mismo tiempo en la posible disminución del desempleo juvenil pues proporciona desde su inicio la experiencia que requieren los empleadores de todo aquel candidato a ocupar una posición dentro de su empresa. Iniciando con una 
revisión de las contribuciones de la educación dual este articulo presenta los inicios del concepto en sí, pasando por la exposición de este en diferentes países y los inicios de este en México, encontrándose estos limitados a la literatura, por lo que se exponen recomendaciones y conclusiones sobre su futura aplicación en este país.

Palabras clave: Educación dual, formación profesional, competencias

\title{
Dual Education : The Analysis and Development of the German Model for its Implementation in the Work Environment
}

\author{
Ibarra Mota Miriam \\ Bribiescas Silva Francisco Arturo \\ Departamento de Ciencias Sociales y Administración, \\ Universidad Autónoma de Ciudad Juárez, Mexico
}

\begin{abstract}
The present article makes an investigation of the German dual education system, as well as its composition, implementation and results, likewise it presents some cases of development in other countries and how this has influenced in some educational systems around the world. Education is a key factor for the development of every country, because it plays a critical role in the productivity of their markets and in their economic growth, because of this the appropriate designs of educational models from their most basic level to its superior aspect, as well as providing a functional vocational orientation to all those who are close to entering professional training. This way, dual education is presented, a system proven over the years in Germany and other European countries to train their workforce within their field of work, interacting in their environment to apply what they have learned. This article will analyze, through non-experimental explanatory research, the development and implementation of the German dual education system and how it has impacted on youth unemployment in their native country, as well as the cases registered within Mexico and what their results have been to the date. For this, several sources of information corresponding to cases mentioned in these countries will be consulted. The concept of dual education was born to respond to the need to adapt Vocational Training to the real needs of the labor market, forming competent professionals within their field so that
\end{abstract}


they contribute actively in different areas of the industry, whether public or private. private, being competent and productive in their specialty, while having an impact on the possible decrease in youth unemployment since it provides from the beginning the experience required by the employers of all that candidate to occupy a position within their company. Starting with a review of the contributions of dual education this article presents the beginnings of the concept itself, going through the exhibition of this in different countries and the beginnings of this in Mexico, being these limited to literature, so they are exposed recommendations and conclusions about its future application in this country.

Keywords: Dual education, professional formation, competences

\section{Introducción}

La educación es un factor clave para el desarrollo de todo país, pues juega un papel crítico en la productividad de sus mercados y en su crecimiento económico, debido a esto es de suma importancia los adecuados diseños de los modelos educativos desde su nivel más básico hasta su aspecto superior, así como el proporcionar una orientación vocacional funcional a todos aquellos que se encuentren próximos a entrar a la formación profesional. Sin embargo, la educación en el aula no puede tomarse como garantía a la correcta formación superior, pues como bien es sabido la práctica lleva a la perfección, es por ello por lo que es necesario integrar la dualidad dentro de la formación profesional para de esta manera aplicar de manera activa los conocimientos que se adquieran de la teoría.

El concepto de educación dual nace para dar respuesta a la necesidad de adecuar la Formación Profesional a las necesidades reales del mercado laboral, formando profesionales competentes dentro de su mercado para que estos contribuyan de manera activa en las diferentes áreas de la industria ya sea pública o privada, siendo competentes y productivos en sus campos de especialidad repercutiendo al mismo tiempo en la posible disminución del desempleo juvenil pues proporciona desde su inicio la experiencia que requieren los empleadores de todo aquel candidato a ocupar una posición dentro de su empresa.

Para infortunio del sistema educativo Mexicano, este solo proporciona la teoría dentro de las aulas, dejando en manos del alumnado la búsqueda de la práctica, sin asegurarse que esta, en conjunto con los conocimientos adquiridos en clase, están ligados entre sí y con los requerimientos del mercado.

Dentro del presente artículo se analizará el desarrollo e implementación del sistema de educación dual alemán y como este ha 
impactado en el desempleo juvenil en su país natal, así como los casos registrados dentro de México y cuales han sido sus resultados a la fecha.

\section{Conceptos}

En este artículo se presentan conceptos como educación dual, formación profesional y sistema educativo los cuales necesitan ser aclarados para el correcto entendimiento de este.

Educación tiene su raíz en la palabra latina ducere (educare, educere). Ducere viene de la raíz indoeuropea deuk, que significa guiar, que a su vez tiene un significado similar a la palabra griega pedagogo, de paidos (niño) y agogos (que conduce), dando a entender que la palabra educación significa guiar o conducir, proporcionar los conocimientos y habilidades necesarias para llegar a un fin, encauzar a alguien al desarrollo de sus posibilidades. La educación se puede definir como un proceso de transmisión de conocimientos y contenidos destinado a desarrollar todas las potencialidades de un individuo, o como la instrucción por medio de la acción docente.

La palabra dual viene del latín dualis (que tiene dos características), sus componentes léxicos son $d u o$ (dos), mas el sufijo al (relativo a). En si la palabra dual hace alusión al conjunto de dos cosas entrelazadas, en un carácter de cualidad.

Lo cual describe a la educación dual como la manera de conducir a alguien a través de los conocimientos y habilidades necesarias para un fin dentro de cualidades entrelazadas relativas a su campo de aplicación en dos áreas, la teoría y la práctica. La dualidad, academia y empresa, ubica el principio fundamental de este hecho educativo admitiendo a la segunda como una nueva escuela, donde el estudiante aprende por medio de la práctica en situaciones o problemas reales de un puesto de trabajo y mediante la aplicación de principios teóricos logra la transformación de la realidad. (Araya Muñoz, 2008)

Por su parte formación, viene del latín forma (figura o imagen), en adición el sufijo -cion indica acción y efecto, por lo cual es la acción y efecto de formar. Profesional también tiene raíces latinas y significa "relativo a la acción y efecto de ejercer un oficio", sus componentes léxicos son el prefijo pro- (a la vista, a favor, adelante), fateri (admitir, confesar), -sio (sufijo que indica acción y efecto), más el sufijo - al (relativo a).

La formación profesional es la acción y efecto de formar a un individuo en lo relativo a un oficio.

Competencia viene del latín competere, aspirar, ir al encuentro de, buscar o pretender algo al mismo tiempo que otros, el adjetivo competente hacer referencia a quien desenvuelve con eficacia un determinado dominio de una actividad, conoce cierta ciencia o materia o experto en las cosas que se expresa o a la que se refiere como competente. 
Las competencias constituyen los comportamientos que ponen en evidencia la capacidad de una persona para movilizar y conjugar sus conocimientos, experiencias, disposiciones, habilidades, actitudes y valores, a fin de abordar, resolver o actuar frente a situaciones del mundo personal, ciudadano, profesional y social. (Camperos Camero, 2008)

\section{Educación dual y sus contribuciones}

La formación profesional alemana goza de una excelente reputación internacional. Según muchos expertos, esta es la razón del desempleo juvenil comparativamente bajo en Alemania. Aunque cada vez más jóvenes eligen estudiar después de la escuela, muchos debates educativos actuales giran en torno a la universidad como lugar de educación. Sin embargo, más de la mitad de las personas todavía comienza una formación profesional dual clásica y la mayoría de la población activa alemana no ha estudiado, pero ha adquirido una cualificación profesional.

Mucho antes de que la industrialización comenzara en Europa, ya había primeros indicios de una formación profesional ordenada en las ciudades. Los gremios como asociaciones profesionales de artesanos (por ejemplo, Weber, panadero, zapatero, herrero) regularon la formación práctica y aprobaron los exámenes finales. Los aprendices eran enseñados por el maestro en una determinada profesión y participaban en la vida familiar del hogar principal; la capacitación en aprendizaje era, por lo tanto, una especie de "educación vocacional" (Stratmman, 1993). Además, la mayoría de las profesiones solo podían practicarlas los miembros del gremio apropiado, es decir, los aprendices, oficiales y amos (BpB, 2016).

Estos gremios surgen durante el siglo XII, y son los antecesores de las actuales cámaras de formación, fueron estos quienes estructuraron el modelo de formación "aprendiz - oficial - maestro" y crearon la especialización en los oficios artesanales. Los maestros tomaban un aprendiz y los enseñaban en un oficio específico, de igual manera se les daba una participación en la vida familiar de la Casa Maestra; el aprendizaje se convirtió en una especie de "formación profesional" (Stratmman, 1993). En ese entonces, como hoy en día, la mayoría de las profesiones solo pueden ser ejercidas por miembros del gremio correspondiente, es decir, por los aprendices, los oficiales y los maestros.

La formación dual alemana comienza en el siglo XIX, con Wilhelm Von Humboldt, Ministro de Educación, quien en 1809 tomo el cargo de dirigir la reforma educativa de Prusia, siendo el principio de este planteamiento la creación de un centro de investigación y enseñanza en Berlín para alumnos y profesores, naciendo de esto el principio oficial de la educación para el trabajo (Ebner, 2016). 
En la segunda mitad del XIX, las primeras empresas industriales introdujeron la formación laboral y fundaron talleres de aprendices. Con el nacimiento del reglamento industrial, en 1869, se introdujo la enseñanza laboral obligatoria para los trabajadores menores de 18 años, donde tenían que aprender a escribir, leer y hacer cuentas, en ese tiempo fueron superadas las ideas económico-liberales que confirieron a la formación profesional la libre capacidad de negociación entre el maestro y el aprendiz, este reglamento estructuró la formación de aprendices de Prusia y en el imperio alemán; la mayor parte de la reglamentación puesta en marcha de la formación fueron traspasados, bajo control estatal, a las camaras, que son los órganos autónomos administrativos del sector privado. Paralelamente se crearon en cada uno de los estados del imperio alemán estructuras jurídicas que reglamentaron las escuelas profesionales; de esa manera se sentaron las bases del sistema de formación actualmente llamado Sistema Dual (Lauterbach, 1997)

La ley de protección del artesanado Handwerkerschutzgesetz de 1897 abrió el camino a un nuevo marco legal. Entre otras cosas se incluyó en la ley el principio dual de formación laboral, es decir, la formación práctica en la empresa junto a las clases teóricas en la escuela, a partir de ese momento, la formación estuvo regulada de manera uniforme en toda Alemania, y el segundo lugar de aprendizaje, junto con la empresa, la escuela vocacional estatal, estaba legalmente anclado.

A principios del siglo $\mathrm{XX}$, la industrialización trajo consigo la necesidad de desarrollar las competencias comerciales, de manera que las empresas introdujeron las profesiones comerciales. El concepto "Escuela de formación" (Berufsschule), aparece en Prusia en 1923.

A partir de la integración europea surge la necesidad de contar con profesionales mejor formados y se exigió que la formación superior no universitaria contara con mayor integración entre teoría y práctica, menor tiempo de estudio y participación de las empresas en la formación curricular construyendo con esto formación profesional para la demanda real. Finalmente, el estado como otro actor central en la educación y formación profesional junto con el sector empresarial (Greinert, 1995) aprobó la Ley de artesanía de 1953 y la Berufsbildungsgesetz Ley de Formación Profesional (BBiG).

Con esta ley, Berufsbildungsgesetz, las diferencias regionales se unificaron en una regulación común. Así, la ley pretendía recoger las necesidades del Estado, los empresarios y los trabajadores.

Educación Dual es un concepto nacido de la necesidad de contar con personal especializado en su área de trabajo, mediante una correcta capacitación, con la educación para el trabajo, es una relación directa y coordinada entre la teoría, que se enseña en las aulas, y la práctica, que se lleva a cabo en las empresas, generando una convergencia didáctica teoría - práctica 
(Escuela-Empresa), y garantizando el desarrollo no sólo de las competencias profesionales del estudiante, sino también las habilidades de análisis crítico, creatividad y visión innovadora.

En el año 1973 se creó la primera Berufsakademie (Academia Profesional) en la ciudad de Stuttgart por parte de las empresas Bosch, Daimler Benz y SEL.

Este "sistema de entrenamiento dual" que se establece hoy en Alemania también es común en Suiza, Austria y Dinamarca (Ebner, 2016). Otros sistemas de educación vocacional y formación ideal son el modelo liberal de "formación en el trabajo" en inglés, en el que las habilidades profesionales se transmiten en la empresa de acuerdo con las necesidades de la empresa.

El desarrollo de este sistema ha permitido que más países se muestren interesados en implementarlo dentro de su cultura educativa, la practicidad del método lo hace un objetivo de copia en muchos sistemas educativos o empresas del sector industrial. Su famosa reputación de ataque al desempleo se ha vuelto una esperanza para países latinoamericanos en los que estas cifras parecen difíciles de disminuir.

El sistema educativo de un pais, sin duda nos aporta mucha informacion sobre el mismo. Hay muchos elementos comunes entre los sistemas educativos europeos, pero cada uno de ellos nos ofrece las singularidades y la manera de ser de sus territorios (Pont, 2015).

De acuerdo con los expertos, los trabajadores calificados alemanes son la base de una producción técnicamente madura y de alta calidad y del éxito de Alemania en la fabricación y exportación de productos de alta calidad (Streeck, 1991). Una característica importante del sistema de educación dual son sus dos lugares de aprendizaje: la escuela vocacional y la empresa. Esto asegura que el aprendizaje teórico se combine con el aprendizaje práctico y la experiencia profesional, es decir, esto facilita que los conceptos aprendidos en el aula sean aplicados correcta y directamente en su campo dentro de la empresa. La mayor parte de la capacitación se lleva a cabo entre tres y cuatro días a la semana en la empresa. La capacitación comienza con un contrato de aprendizaje, que se celebra entre el aprendiz y la empresa, durante el período de capacitación, que generalmente toma tres años. Al finalizar los exámenes finales hay un certificado de la escuela profesional. Los alumnos también reciben remuneración, lo que aumenta con la formación profesional avanzada y también es de suma importancia para algunos graduados de la escuela secundaria para optar por un estudio.

Otra característica especial de la educación dual es la igualdad de participación del Estado y la economía: los conjuntos de estado a través de la Berufsbildungsgesetz (Ley de formación profesional) y Ley del Artesano y las normas para la formación establecida (derechos y obligaciones de Becarios, 
remuneración, perfil del personal de formación y capacitación, arreglos para exámenes finales, etc.) y la economía se ve por su parte en la formación profesional de los agentes sociales - estas son las asociaciones de empresarios y sindicatos - determinar el contenido y las condiciones para las ocupaciones individuales. El Instituto Federal de Formación Profesional (BIBB) prepara reglamentos de formación y desarrolla en conjunto con expertos de la práctica profesional, nombrados de esta manera por los agentes sociales, los reglamentos del proyecto de educación; también se elaboran, en conjunto con los profesionales expertos, los programas de educación profesional y sus programas para la correcta coordinación entre lo enseñado dentro de las aulas y lo implementado en las empresas, esta tarea corre por parte de los Ministerios de Educación.

La inclusión de la economía asegura que la capacitación proporcione los conocimientos y habilidades que realmente se necesitan en la vida laboral. La cooperación del estado a través del $\mathrm{BBiG}$, la ley del artesano y las normas de formación, a su vez, garantiza que las empresas en Alemania tengan que ceñirse a reglas claras durante el entrenamiento. Esta "estandarización" de la capacitación en todas las empresas crea una clara ventaja sobre otros sistemas de capacitación. En Alemania, se mantienen los estándares de calidad y se desarrollan competencias, que son importantes para la economía en su conjunto y no solo para una empresa. Las personas con un título de doble educación también pueden usar sus conocimientos adquiridos en diferentes compañías y cambiar de una a otra más fácilmente $(\mathrm{BpB})$.

\section{Marco referencial}

El Sistema de Educación Dual es una Formación Profesional que combina el aprendizaje en una compañía y en una berufsakademie (Centro de formación profesional). Este sistema es practicado en diversos países, especialmente en Alemania, Austria, Bosnia y Herzegovina, Croacia, Serbia, Eslovenia, Macedonia, Montenegro, y Suiza; pero también en Dinamarca, Países Bajos y Francia. El término "Dual" indica que son dos los sujetos responsables de la enseñanza, y el término "sistema" indica que ambos no son independientes entre sí, sino que actúan conjuntamente (Semmel, 1989). Esto permite la interacción de los aprendices con el mercado laboral en un ambiente controlado y asesorado por tutores que, de igual manera, tienen una formación especial que les permite vincular y evaluar las actividades que se realizaran en la empresa con los conocimientos teóricos que se adquieren en el aula, para de esta manera formar personal que cumpla con los requerimientos de las empresas y sus posiciones.

Este sistema es aplicado a nivel técnico en Alemania, teniendo un alto reconocimiento, y cuenta con validez como formación profesional para aquellos que decidieron no continuar con su formación universitaria, al igual 
que para aquellos que debido a dificultades económicas no pudieron continuar con su educación superior. Como se mencionó anteriormente, debido a que la formación profesional Alemana tiene lugar en dos sitios de aprendizaje se le denomina dual. Así también las normas, la financiación y la administración del aprendizaje en la escuela, y en la empresa se encuentran bajo diferentes demarcaciones (Lauterbach, 1997). Un ejemplo es la carga de horas establecida para cada parte, la cual es determinada por los estados federados o Länder, que estipulan un total de entre ocho a doce horas, por semana, para que los aprendices asistan a tomar clases mientras que el resto de las horas, generalmente manejado a un 60 o 70\%, con dedicados a recibir formación dentro de una empresa, cuyo contenido es señalado en los planes marco de enseñanza establecidos para la profesión elegida, que a su vez son diseñados de acuerdo a lo establecido por el Ausbildungsordnung (Reglamento de Formación). Cada Länder elabora sus planes de enseñanza conforme a lo establecido en el plan marco, generando así que una misma profesión tenga diferentes planes de estudio de acuerdo con el estado en el que se viva, aunque con el tiempo se ha llegado a generalizar el contenido del plan conforme a la carrera, es decir, las variaciones de los planes para una profesión demandada y general son mínimas.

Falcon (2015) señala que tanto los reglamentos como el diseño de los planes de educación y enseñanza se construyen conjuntamente en una participación paralela entre los interlocutores sociales y el gobierno federal, en un lado, y la conferencia de los ministros de educación de otro, con la finalidad de concretizar los puntos de interés de cada uno y así gestar un marco general a nivel federal.

Velarde y Medina (2014) mencionan que en la actualidad los sistemas educativos, afrontan dos grandes retos íntimamente relacionados: por un lado, consolidar una escuela comprensiva que permita el máximo desarrollo de las capacidades de cada persona, respetando la diversidad y asegurando la equidad de acceso a la educación. Compensando las desigualdades, favoreciendo la formación de sujetos autónomos, capaces de tomar decisiones informadas sobre su propia vida. Participando de manera relativamente autónoma en la vida profesional y social.

Martinez, Navarro y Sanchez (2012) señalan que la dificultad de la formación del sujeto autónomo está situada en el reto de convertir las información en un conocimiento que lo guie a tener una mejor comprensión de la realidad y que de esta manera el conocimiento pase a ser pensamiento y sabiduría, conectando el contexto social e histórico con los conocimientos adquiridos en la escuela, es decir, formar una competencia que le permita entender la situación y de esta manera sintetizar todo aquello aprendido con el fin de aplicarlo al reto presentado, es decir, desarrollar competencias. Según la Organización Internacional del Trabajo, la competencia laboral incluye 
conocimientos generales y específicos, habilidades y calificaciones que le permiten a la persona desempeñarse correctamente, de acuerdo con el resultado esperado y con capacidad para resolver con éxito situaciones inciertas, nuevas e irregulares (Álvarez, 2004). El aprendizaje pasaría a ser una experiencia práctica que permite el desarrollo de las competencias y no sólo una experiencia basada en la abstracción y en la discusión teórica.

En los últimos 20 años la economía mundial ha pasado por enormes transformaciones, los sistemas educacionales han pasado a tomar la calidad de estos como un factor de peso en la prosperidad de la economía de una nación. Estas transformaciones, son sustentadas mediante la revolución de telecomunicaciones e información, y requieren una infraestructura que incluye, entre otros elementos, una fuerza de trabajo con una sólida base de conocimientos matemáticos y científicos y una capacidad para comunicarse eficazmente. (Carnoy \& Castro, 1997)

Con el propósito de lograr una sinergia entre mercado y educación que haga frente a estas transformaciones Alemania ha sido el principal impulsor de este programa educativo, pero alrededor de mundo se han sumado a su implementación, adaptándolo siempre a su cultura. Desde Europa, hasta Latinoamérica, la educación dual se ha involucrado en diferentes modelos educativos.

En la Comunidad de Madrid, en el año 2011, se pusieron en marcha proyectos de formación profesional dual en dos ciclos formativos: Desarrollo de Aplicaciones Informáticas y Mantenimiento Aeromecánico, ambos de grado superior (Rego Agraso, Cerqueiras, Eva, \& Rial Sánchez, 2015). Fueron las propias empresas las que, en colaboración con el centro de formación profesional, se encargaron de organizar todo el proceso de enseñanzaaprendizaje, además de seleccionar y conceder becas al alumnado. Éste permaneció dos tercios del tiempo destinado a la formación en la propia empresa, ampliando así de forma comparativa a la duración del módulo de Formación en Centros de Trabajo (FCT) en un ciclo formativo ordinario. En Galicia, destaca el proyecto experimental puesto en marcha en el año 2013 entre la empresa Coremain SLU6 y el Centro Integrado de Formación Profesional Politécnico de Santiago de Compostela. El modelo dual se desarrolló en el ciclo formativo de grado superior de Sistemas Informáticos y de Telecomunicaciones con un grupo inicial de 15 alumnos/as. En esta ocasión, un 21,7\% del tiempo de duración del ciclo se llevó a cabo en el centro formativo, mientras que el tiempo restante se desarrolló en la propia empresa.

Hoeckel, Field y Grubb, a través de la OCDE (2009) señalan que los programas VET (Vocational Education and Training) se proporcionan principalmente en lo que Suiza se refiere a un enfoque dual de aprendizaje, que combina estudios a tiempo parcial en una escuela vocacional y pasantías a tiempo parcial en una empresa de acogida. En comparación internacional, 
Suiza se destaca en términos de la participación de la formación profesional en el nivel secundario superior y el número de aprendices como un porcentaje de los que tienen entre 15 y 64 años; otros países ofrecen una formación profesional mucho menos práctica.

Los programas de formación profesional en Suiza suelen tener lugar en tres diferentes lugares de aprendizaje / capacitación: los estudiantes de formación profesional pasan parte de su tiempo en una escuela vocacional; parte de su tiempo haciendo un aprendizaje en una empresa anfitriona; y para la mayoría de VET programas, los estudiantes de formación profesional asisten a cursos de la industria en un centro de capacitación industrial para desarrollar habilidades prácticas complementarias relacionadas con la ocupación en cuestión. El balance de los componentes basados en el aprendizaje y los basados en la escuela varían, pero se decide por acuerdo tripartito y escrito en las ordenanzas de formación profesional.

Los centros de FP Holandeses se caracterizan por ser macrocentros regionales que aglutinan grandes cantidades de alumnos y lideran procesos de innovación y actualización de contenidos junto a agrupaciones empresariales. (Alianza Formación Profesional Dual). Las cualificaciones medias vinculadas a la formación profesional son las más frecuentes entre la población ocupada de Holanda, pues el $40 \%$ de los trabajadores holandeses tiene este nivel de formación. Entre los alumnos de formación profesional, un 55\% optan por un itinerario dual, que se puede implantar de dos formas diferentes: un itinerario dual basado en la empresa con una formación en la empresa de entre el $60 \%$ y $70 \%$ o un itinerario basado en la escuela, en el que un $25 \%$ de los contenidos se reciben en la empresa.

El sistema educativo de Japón se reconoce como uno de los más exitosos del mundo, no solo por su éxito académico o por los resultados de aprendizaje obtenidos en los informes PISA, sino también por el ajuste entre las habilidades formadas y las habilidades requeridas por el mercado laboral, es decir, por la cualificación de la fuerza productiva de (de Bogotá, 2015), por lo que ha sido referente para el sistema educativo de muchos otros países. Actualmente, no obstante, el $25 \%$ de los estudiantes acuden a los senmongako, donde tras obtener un diploma en dos o tres años, pueden optar a la universidad, o bien continuar dos años más de FP y obtener un diploma advanced. Aunque las escuelas especialistas de FP o specialised higher education mantienen en su currículo materias genéricas, como lengua japonesa, matemáticas y lengua extranjera, de un $30 \%$ a un $80 \%$ de los créditos se dedican a materias vocacionales. El incremento de estudiantes en estos centros se explica porque esta opción de educación terciaria responde mejor a las demandas del sector empresarial y, por tanto, ofrece mayores posibilidades de empleo. 
Específicamente, en la Argentina, los currículos por competencias emanan desde sus correspondientes ministerios de educación bajo la forma de perfiles profesionales contenidos en catálogos nacionales de programas de Educación Técnica Profesional (ETP) tanto de nivel secundario como superior. (Sevilla \& Paola, 2017). Dichos programas son impartidos y certificados a nivel técnico, el Centro de Capacitación Profesional (BBZ) y la Cámara de Industria y Comercio Argentino-alemana, en representación de las empresas capacitadoras, son los responsables de la capacitación de los postulantes de los países Argentina. La teoría y la capacitación general se llevan a cabo principalmente en el Centro de Capacitación Profesional en Buenos Aires (BBZ). Por el otro lado, la práctica laboral se lleva a cabo en las empresas capacitadoras de Argentina, Uruguay o bien en Paraguay. Ellas, juntamente con el Centro de Capacitación Profesional, son los responsables de la capacitación de futuros jóvenes profesionales. La capacitación tiene una duración de 2 años, las clases teóricas en el BBZ se dividen en 8 bloques de 4-5 semanas de duración cada una. Luego de cada bloque en el BBZ, se realiza una intensiva práctica en la empresa internacional argentino-alemana.

Por otra parte, en México, cuestionándose cada vez más el sistema tradicional de enseñanza, requiere que todos los jóvenes puedan tener acceso a una educación superior de calidad, los contenidos y métodos educativos deberían responder a las características demandadas por el mercado laboral. Requiriéndose plantear nuevas prácticas activas, basadas en el logro de competencias que tengan en cuenta el proceso de enseñanza-aprendizaje.

En otras palabras, para lograr una mejor integración laboral de los profesionistas mexicanos, es necesario trabajar en conjunto con los actores de su entorno, es decir, sociedad, empresas, escuelas y el propio individuo, y lograr una sinergia en un modelo que conduzca a los cuatro miembros a lograr sus objetivos dentro del entorno, para de esta manera impulsar el crecimiento del lugar donde este se implemente. Desde la perspectiva administrativa, el ciclo de Deming es necesario en toda implementación e integración dentro de un proyecto, así como la dinamicidad, la flexibilidad y el enfoque en un plazo específico para su concepción, desarrollo, evaluación y control.

México se encuentra, desde 2013, desarrollando sus primeros intentos en la adaptación del sistema de educación dual. La Secretaría de Educación Pública (SEP) a través de la Subsecretaría de Educación Media Superior (SEMS) en coordinación con la Confederación Patronal de la República Mexicana (COPARMEX) y con la asesoría de la Cámara México - Alemana de Comercio e Industria (CAMEXA) y la experiencia en el tema del Colegio Nacional de Educación Profesional Técnica (CONALEP), desarrollaron en 2013 el Modelo Mexicano de Formación Dual (MMFD), el cual retoma los elementos esenciales del modelo dual alemán y los adapta a la realidad nacional. 
A partir de los aprendizajes generados y con el propósito de conceptualizar e institucionalizar a la formación dual en el nivel medio superior, el 11 de junio de 2015 se publicó en el Diario Oficial de la Federación el Acuerdo Secretarial número 06/06/15 por el que se establece, caracteriza y regula a la formación dual como una opción educativa del tipo medio superior.

Esta nueva opción educativa pertenece a la modalidad mixta y se caracteriza porque las actividades de aprendizaje previstas en el plan de estudios se desarrollan tanto en las instituciones educativas que lo ofrecen, como en contextos reales de aprendizaje mediante trayectos curriculares flexibles. Lo anterior, permitirá a los estudiantes desarrollar conocimientos y competencias en las empresas; vincular de manera temprana y simultánea la teoría y la práctica educativa para fortalecer el desarrollo integral de sus habilidades; así como contribuir a mejorar su empleabilidad. (SEP, 2016)

El Modelo Mexicano de Formación Dual pretende que los estudiantes obtengan una formación más pertinente, que deberá traducirse, para las empresas, en mayores niveles de productividad y menor rotación del personal calificado, menores tasas de desempleo y mas ingresos para los jóvenes egresados. Esto implicara para los planteles escolares una renovación permanente de sus planes y programas de estudios, así como la colocación exitosa de sus egresados. (Jiménez, Salinas, \& Jaime, 2016)

\section{Resultados y recomendaciones}

Como se ha estudiado a lo largo de este artículo, la formación profesional llevada de la mano por la educación dual, ha dejado muchos beneficios a lo largo de diferentes países, desafortunadamente hasta el momento, los datos existentes sobre educación dual en México se han limitado a literatura, por lo que se recomienda realizar una investigación de la cual se obtengan datos duros sobre los cuales se pueda crear un modelo de educación dual que pueda aplicarse a la cultura y sistema educativo de México, así como crear un estándar sobre el cual se puedan construir las carreras de los profesionistas futuros que podrán desarrollar mejor la industria para el país, así como elevar la productividad del sector en el que se aplique.

\section{Conclusion}

El hecho real es que la educación sólo constituye un factor, aunque de mucha importancia, en el conjunto de elementos y condicionantes que determinan, a nivel sociedad, la productividad y la competitividad económicas, así como los niveles de criminalidad, la participación política, los niveles de salud, etcétera. La educación ejerce efectos potenciales muy poderosos en cada una de esas áreas, si las condiciones necesarias y los insumos requeridos se encuentran presentes (Hernández Laos, 2004). De esta manera, involucrando a la educación mexicana en un proceso de mejora 
continua, como al que debe someterse todo aquello que sea medible, se puede contribuir a la mejora continua de nuestra sociedad, y, por lo tanto, de nuestro país.

A lo largo de las décadas se ha demostrado que el modelo de educación dual influye en el desarrollo de los profesionistas, ya sea mediante una formación técnica o administrativa, y que el mismo modelo ha sido capaz de adaptarse a distintas naciones bajo un esquema de formación bien definido que ha podido lograr que potencias mundiales como Alemania y Japón cuenten con personal competente y dedicado en y a su labor. La reciente adaptación de este método en la república mexicana podría llegar a ser la base para que se alcance un bien mayor, ya que actualmente se encuentra desplegado únicamente a nivel medio superior.

Lograr la adaptación y aceptación de este modelo en nivel superior podría traer beneficios dentro de varias áreas, y, sobre todo, beneficiaria el nivel industrial de esta frontera y sus profesionistas, dada la naturaleza de este territorio la influencia de una formación profesional bien estructurada $\mathrm{y}$ fundamentada, así como su seguimiento en profesiones de naturaleza industrial podría fortalecer la inserción laboral de los individuos recién egresados.

\section{References:}

1. Álvarez, R. P. (2004). Formación superior basada en competencias, interdisciplinariedad y trabajo autónomo del estudiante. Revista iberoamericana de educación, 35(1), 1-33.

2. Araya Muñoz, I. (2008). La formación dual y su fundamentación curricular. Educación, 32(1).

3. BpB. (2016). Bundeszentrale fur politische Bildung.

4. Camperos Camero, M. (2008). La evaluación por competencias, mitos, peligros y desafíos. Educere, 12(43), 805-814.

5. Carnoy, M., \& Castro, C. d. M. (1997). ¿ Qué rumbo debe tomar el mejoramiento de la educación en América Latina? Retrieved from

6. de Bogotá, C. d. C. (2015). Informe estadístico de consultas del CIEB del 2015.

7. Ebner, C. U., Alexandra. (2016). Entstehung und Merkmale des dualen Ausbildungssystems.

Retrieved from http://www.bpb.de/gesellschaft/kultur/zukunft-bildung/230376/dualeausbildung

8. Falcón, J. A. A. (2015). El sistema dual de formación profesional alemán: escuela y empresa. Educação e Pesquisa, 41(2), 495-511.

9. Greinert, W.-D. (1995). Educacion y formacion profesional de masas en Europa. Cedepof. Cedepof. 
10. Hernández Laos, E. (2004). Panorama del mercado laboral de profesionistas en México. Economía unam, 1(2), 98-109.

11. Hoeckel, K., Field, S., \& Grubb, W. N. (2009). Learning for Jobs: OECD Reviews of vocational education and training: Switzerland. Organisation for Economic Co-Operation and Development. April, Paris.

12. Jiménez, E. A., Salinas, M. C., \& Jaime, E. T. (2016). Resultados obtenidos en el sector empresarial del modelo de eduacion dual COPARMEX-EDOMEX-OTE. ANFEI Digital(4).

13. Lauterbach, U., \& Lazendorf, U. (1997). El sistema Dual de formacion profesional en Alemania: Funcionamiento y situacion actual. Revista Universitaria de Formacion Profesional, 51-68.

14. Martínez, A. M., Navarro, J. G. C., \& Sánchez, J. A. R. (2012). Aprendizaje basado en competencias: una propuesta para la autoevaluación del docente. Profesorado. Revista de currículum y formación de profesorado, 16(2), 325-338.

15. Mundial, B. OCDE (2012). La Educación Superior en Colombia.

16. Pont, M. B. (2015). El sistema educativo de Alemania. Avances en Supervisión Educativa.

17. Rego Agraso, L., Cerqueiras, B., Eva, M., \& Rial Sánchez, A. F. (2015). Formación profesional dual: comparativa entre el sistema alemán y el incipiente modelo español. Revista Española de Educación Comparada, 25, 149-166.

18. Semmel, H. H. (1989). Formación profesional dual.

19. SEP. (2016). Modelo Mexicano de Formacion Dual. Retrieved from http://www.sems.gob.mx/es_mx/sems/modelo_mexicano_formacion dual

20. Sevilla, B., \& Paola, M. (2017). Panorama de la educación técnica profesional en América Latina y el Caribe.

21. Stratmman, K. (1993). La educación de aprendiz comercial en Alemania. Historia de la modernización de la formación profesional. Formación profesional en la Sociedad Estatal (1648-1806), 1.

22. Streeck, W. (1991). Sobre las acciones sociales y politicas de la produccion de calidad diversificada.

23. Velarde, L., \& Medina, A. (2014). Sistema Dual de Aprendizaje: Un modelo educativo dinámico para una formación dual. Paper presented at the Educación. Handbook T-IV: Congreso Interdisciplinario de Cuerpos Académicos. 